\title{
SOME NO-ARBITRAGE RULES UNDER SHORT-SALES CONSTRAINTS AND APPLICATIONS TO CONVERGING ASSET PRICES
}

\author{
DELIA COCULESCU AND MONIQUE JEANBLANC
}

\begin{abstract}
Under short sales prohibitions, no free lunch with vanishing risk (NFLVRS) is known to be equivalent to the existence of an equivalent supermartingale measure for the price processes (Pulido [26]). We give a necessary condition for the drift of a price process to satsify (NFLVRS). For two given price processes, we introduce the concept of fundamental supermartingale measure, and when a certain condition necessary to the construction of this fundamental supermartingale measure is not fulfilled, we provide the corresponding arbitrage portfolios. The motivation of our study lies in understanding the particular case of converging prices, i.e., two prices that coincide at a bounded random time.
\end{abstract}

\section{INTRODUCTION}

In arbitrage-free financial markets, the law of one price simply states that similar financial assets, i.e., that have identical payoffs, should be sold at the same price in different locations. There are some particular assumptions about the financial markets that lead to this fundamental result, importantly investors need to be able to observe the prices in the different locations and to sell short the corresponding assets. Also, there should be no transaction costs. Indeed, under these assumptions, any investor is able to construct an arbitrage portfolio consisting in a short position in the (relatively) overpriced asset and a long position in the (relatively) underpriced asset, thus making a riskless profit. This represents the simplest arbitrage strategy one can encounter: not only is it a buy and hold strategy, but additionally, it is model independent, i.e., does not rely upon an underlying model for describing the prices dynamics in time.

Obviously, in case of short sales prohibitions, the above described arbitrage portfolios are impossible to construct, hence similar assets may have differing prices: the rule of one price does not apply. A question arises naturally: How may the differing prices behave as stochastic processes within the limits of no arbitrage with short sales constraints? The aim of this paper is precisely to shed light on this question.

With this motivation in mind, we study the probabilistic properties of two stochastic processes when one imposes the no free lunch with vanishing risk condition under short sales constraints, abbreviated (NFLVRS). This condition was introduced by Pulido in [26], as the counterpart -when investors are not allowed to short sell- of the no arbitrage paradigm (NFLVR) of Delbaen and Schachermayer (see [8] and [10]). For the reader's convenience, 
the definition of (NFLVRS) is provided in Section 2, Based on previous work by Jouini and Kallal [19], Fritelli [14], Pham and Touzi [25], Napp [22] and Karatzas and Kardaras [20], the paper by Pulido [26] establishes important properties of price processes under short sale prohibitions namely the equivalence between (NFLVRS) and the existence of an equivalent supermartingale measure for the price processes. In the current paper, we shall rather translate the condition (NFLVRS) in terms of "structure conditions under short sales constraints" for the underlying stochastic processes and define the notion of fundamental supermartingale measure. When a certain condition, necessary to the construction of the fundamental supermartingale measure is not fulfilled, we provide the corresponding arbitrage portfolios.

The developed theory is illustrated with many examples of converging asset prices, that is, price processes that are expected to "cross", i.e., to reach almost surely the same value over some bounded horizon, which is the mathematical description of the similar assets. In this particular framework of converging prices, the existence of imperfect and asymmetric information is crucial to justify the formation and persistence in time of the differing prices. This element is integrated in our analysis: we assume that each individual price is formed given some distinct information set (filtration) a priori unrelated with the information set that drives the price formation in a different location, except measurability of the final payoff in both situations. The no arbitrage conditions are analysed from the perspective of an agent (called the insider) that has access to a global information set, i.e., that comprises the observation of the two differing prices. The insider can trade in both markets, but cannot sell short.

There are many examples of converging prices, the simplest being a future contract and its underlying asset, or the two portfolios arising from the call-put parity (i.e., one consisting of a call option and bonds, the second of a put option and underlying stock). In markets with short sales prohibitions, the call-put parity is not expected to hold in every point in time but we observe the identity of the payoffs at maturity. Other examples of convergence are represented by some portfolios that are commonly used in capital structure arbitrages or the pairs trading. Note however that in these cases the convergence is model-based; in capital structure arbitrages a particular "structural" model is assumed to explain the joint evolution of the prices for the different securities with common issuer, while in the pairs trading, the pairs are selected upon a statistical analysis. Nevertheless, assuming that the underlying models are "correct" the question remains the same: how to construct the strategies when selling short is not possible? Finally, our framework applies well to the case of similar derivative contracts that are sold over the counter, and thus differing prices typically arise as a consequence of a imperfect information between the different sellers and buyers.

The remaining of the paper is organised as follows: Section 2 introduces the probabilistic model for the two converging prices and recalls the no arbitrage framework we adopt in this paper. Section 3 establishes the "structure conditions" in Theorem 3.4. In Section 4. we derive sufficient conditions for the existence of a supermartingale measure as well as 
some necessary conditions. We introduce a probability measure that we call fundamental supermartingale measure and arbitrage portfolios are provided when a certain condition is not fulfilled and the fundamental supermartingale measure cannot be constructed. Section 5 analyses many examples of converging prices. Let us emphasise that our main results in Section 3 and Section 4, (in particular the structure conditions in Theorem 3.4, the construction of the fundamental supermartingale measure and the arbitrage portfolios is Theorem 4.2 and Lemma 4.7), are more general: the property of the two prices to be converging is not used for deriving these results.

\section{A Stochastic Model with two Converging Asset Prices}

In this paper, all filtrations are defined on a probability space $(\Omega, \mathcal{F}, \mathbb{P})$.

We consider two financial assets, possibly traded in different locations (e.g. exchanges). Their respective price processes are denoted by $X:=\left(X_{t}\right)_{t \geq 0}$ and $Y:=\left(Y_{t}\right)_{t \geq 0}$, while $\mathbb{F}^{X}:=\left(\mathcal{F}_{t}^{X}\right)_{t \geq 0}$ and $\mathbb{F}^{Y}:=\left(\mathcal{F}_{t}^{Y}\right)_{t \geq 0}$ are their respective right-continuous $\mathbb{P}$-augmented filtrations. The spot interest rates are constant and equal to zero, that is, the price processes $X$ and $Y$ are already discounted. We assume that an investor (called hereafter the insider) is able to observe the price dynamics in the two locations, so that his information flow is given by $\mathbb{G}:=\left(\mathcal{G}_{t}\right)_{t \geq 0}$ with

$$
\mathcal{G}_{t}=\cap_{s>t} \mathcal{F}_{s}^{X} \vee \mathcal{F}_{s}^{Y} .
$$

Also, the insider has a bounded trading horizon, denoted $T$, which is a $\mathbb{G}$-stopping time. Following [26], we suppose that $X$ and $Y$ are nonnegative $\mathbb{G}$ semimartingales with right continuous sample paths that are locally bounded.

Many examples that we're considering fit in the following framework:

Definition 2.1. A couple of financial assets $(X, Y)$ are said to have converging prices if the $\mathbb{G}$-stopping time $\inf \left\{t \in \mathbb{R}_{+} \mid X_{t}=Y_{t}\right\}$ is bounded.

When $X$ and $Y$ are converging prices, we shall consider that the insider's horizon is a given point of convergence of the two prices, i.e., $T$ is such that $T$ is a bounded $\mathbb{G}$-stopping time and such that

$$
\xi:=X_{T}=Y_{T} .
$$

One can take $T=\inf \left\{t \in \mathbb{R}_{+} \mid X_{t}=Y_{t}\right\}$, but such a restriction is not necessary. In some situations the $\mathbb{G}$-stopping time $T$ can be chosen as the maturity of the assets, when the cash flow $\xi$ is paid to the investors that have long positions either in the asset $X$ or $Y$. In this case $T$ should be an $\mathbb{F}^{X}$ and an $\mathbb{F}^{Y}$-stopping time (i.e., cash flows are always observable by holders of long positions in the corresponding assets). Another interesting situation is when $T$ is only observed by the insider, hence $T$ is neither an $\mathbb{F}^{X}$ nor an $\mathbb{F}^{Y}$-stopping time. Either of the two interpretations are possible here, i.e., we do not require $T$ to be more than a bounded $\mathbb{G}$-stopping time, but remaining fixed through the analysis. 
Our aim is to analyse the no arbitrage property (NFLVRS) from the insider's perspective, i.e., when there are prohibitions for the insider to sell short the assets $X$ and $Y$. In other words, we consider that the investor's strategies involve the following positions: long or short in cash $\left(\pi^{C}\right)$ and only long positions in $X$ and $Y\left(\pi^{X}\right.$ and $\left.\pi^{Y}\right)$, consequently the value of the investor's portfolio writes:

$$
V_{t}^{\pi}:=\pi_{t}^{C}+\pi_{t}^{X} X_{t}+\pi_{t}^{Y} Y_{t}
$$

and, when self financing, we have $d V_{t}^{\pi}:=\pi_{t}^{X} d X_{t}+\pi_{t}^{Y} d Y_{t}$. As usual, we impose some admissibility conditions for strategies under (NFLVRS) in this framework. We refer to Pulido [26] for more details.

Definition 2.2. A trading strategy is a $\mathbb{G}$-predictable process $\pi=\left(\pi^{C}, \pi^{X}, \pi^{Y}\right)$. A trading strategy $\pi$ is called an admissible trading strategy under short sales prohibitions for $X$ and $Y$ if:

(i) $\pi^{X} \in L(X)$ and $\pi^{Y} \in L(Y)$ (i.e., $\pi^{X}$ is integrable with respect to the semimartingale $X, \pi^{Y}$ is integrable with respect to the semimartingale $\left.Y\right)$.

(ii) The process $V^{\pi}$ is bounded from below.

(iii) $\pi^{X} \geq 0$ and $\pi^{Y} \geq 0$.

We denote by $\mathcal{A}$ the set of admissible trading strategies under short sales restrictions for $X$ and $Y$.

We now define the following sets:

$$
\mathcal{K}:=\left\{V_{T}^{\pi}, \pi \in \mathcal{A}\right\} \quad \mathcal{C}:=\left(\mathcal{K}-L_{+}^{0}(\mathbb{P})\right) \cap L^{\infty}(\mathbb{P}) .
$$

where $L_{+}^{0}(\mathbb{P})$ is the space of equivalence classes of nonnegative finite random variables, and $L^{\infty}(\mathbb{P})$ is the space of $\mathbb{P}$-essentially bounded random variables. No Free Lunch with Vanishing Risk under short sales prohibition (NFLVRS) is defined as follows: (NFLVRS) holds if $\overline{\mathcal{C}} \cap L_{+}^{0}(\mathbb{P})=\{0\}$, where $\overline{\mathcal{C}}$ is the closure of $\mathcal{C}$ with respect with the $\|\cdot\|_{\infty}$ norm in $L^{\infty}(\mathbb{P})$.

Theorem 2.3. [26] (NFLVRS) holds if and only if there exists a probability measure $\widetilde{\mathbb{P}}$ on $(\Omega, \mathbb{P})$, with $\widetilde{\mathbb{P}} \sim \mathbb{P}$ and such that the processes $X$ and $Y$ are $(\mathbb{G}, \widetilde{\mathbb{P}})$-supermartingales. Such a probability measure $\widetilde{\mathbb{P}}$ is called a supermartingale measure.

Because the condition of no arbitrages in the form of (NFLVRS) is equivalent to the existence of a supermartingale measure for the couple $(X, Y)$ in the filtration $\mathbb{G}$, our aim is to shed light on the properties of processes $X$ and $Y$ when considered as stochastic processes in the larger filtration $\mathbb{G}$, under the requirement that there exists a probability measure $\widetilde{\mathbb{P}}$ such that $\widetilde{\mathbb{P}} \sim \mathbb{P}$ such that the processes $X$ and $Y$ are $(\mathbb{G}, \widetilde{\mathbb{P}})$-supermartingales. 


\section{SOME IMPLICATIONS OF (NFLVRS) ON A PRICE PROCESS}

We aim to investigate the properties of processes that admit an equivalent supermartingale measure. In the classical setting of no arbitrage (without short selling constraints), structure conditions have been defined; they are derived from imposing the existence of a strict martingale density. Roughly speaking, if $X$ satisfies (NFLVR) and some regularity conditions, there exists a process $\lambda$ such that $X=\lambda \cdot\langle M\rangle+M$, with $M$ being a local martingale. We refer to Back [3], Schweizer [27], Föllmer and Schweizer [12], Ansel and Stricker [2], Schweizer [28], Choulli and Stricker [6] for precise details.

We shall carry out our analysis in the filtration $\mathbb{G}$ and the interval $[0, T]$, i.e., the insider's information set and the insider's investing horizon.

To begin with, let us introduce some notation that are going to be used in the remaining of the paper:

Notation 3.1. $\quad$ (i) We write $\langle Z\rangle$ for the predictable bracket of a semimartingale $Z$ under the measure $\mathbb{P}$ and in the filtration $\mathbb{G}$. Whenever the underlying filtration we are considering is not $\mathbb{G}$ and/or the probability is not $\mathbb{P}$ we shall use explicit notations: for instance $\langle Z\rangle^{(\mathbb{F}, \mathbb{Q})}$ is the predictable bracket under a probability measure $\mathbb{Q}$ and in a filtration $\mathbb{F}$ (in that case, $Z$ needs to be an $\mathbb{F}$-semimartingale).

(ii) The expectation operator under the probability $\mathbb{P}$ is written $\mathbb{E}$; whenever the probability measure is a different one, we shall use an explicit notation, i.e., $\mathbb{E}^{\mathbb{Q}}$ is the expectation under the probability measure $\mathbb{Q}$.

(iii) $\mathcal{P}(\mathbb{F})$ is the class of $\mathbb{F}$-predictable processes, where $\mathbb{F}$ is a given filtration.

(iv) $\mathcal{S}(M)$ is the stable subset of $(\mathbb{G}, \mathbb{P})$-local martingales generated by $M$, where $M$ is $a(\mathbb{G}, \mathbb{P})$-locally square integrable martingale; $\mathcal{S}(M)^{\perp}$ is the set of $(\mathbb{G}, \mathbb{P})$-locally square integrable martingales that are strongly orthogonal to $M$.

(v) $\mathcal{E}(Z)$ denotes the Doléans-Dade exponential of a semimartingale $Z$ with $\mathcal{E}_{0}(Z)=$ 0 .

(vi) We use the notation $\{d A \neq 0\}$, or alternatively $\{d A>0\}$, for the support of the measure $d A$, associated to a non decreasing process $A$.

The following result is a more precise formulation of Theorem 2.3 in the particular case of converging prices:

Lemma 3.2. Suppose that $(X, Y)$ are converging prices, that is $X_{T}=Y_{T}=\xi$. Then, the prices $(X, Y)$ satisfy (NFLVRS) if and only if there exists a probability measure $\widetilde{\mathbb{P}}$ such that $\widetilde{\mathbb{P}} \sim \mathbb{P}$ and

$$
\begin{aligned}
& X=\widetilde{M}+\widetilde{Z}^{X} \\
& Y=\widetilde{M}+\widetilde{Z}^{Y},
\end{aligned}
$$

where $\widetilde{M}_{t}:=\mathbb{E}^{\widetilde{\mathbb{P}}}\left[\xi \mid \mathcal{G}_{t}\right]$ and $\widetilde{Z}^{X}$ and $\widetilde{Z}^{Y}$ are two $(\mathbb{G}, \widetilde{\mathbb{P}})$-potentials (i.e., are positive supermartingales satisfying $\widetilde{Z}_{T}^{X}=\widetilde{Z}_{T}^{Y}=0$ ). 
Proof. (NFLVRS) holds if and only if a supermartingale measure $\widetilde{\mathbb{P}}$ exists. But then $X$ and $Y$ are uniformly integrable $\widetilde{\mathbb{P}}$-supermartingales and the expressions follow from the Riesz decomposition and the terminal condition $X_{T}=Y_{T}$. For more details, see [21, VI-11] or, alternatively, [7, T12 p. 97].

Now, we investigate the structure of price processes under the reference probability $\mathbb{P}$, which is arbitrarily chosen. We only detail the case of one of the assets.

Remark 3.3. We have assumed from the start that $X$ is a locally bounded $(\mathbb{G}, \mathbb{P})$ semimartingale, hence it is special [15, Corollary 8.7.].

Below, we also exploit some additional assumption, that is, there exists an equivalent local martingale measure for $X$ when considered as a stochastic process in its own filtration:

$(\mathrm{NFLVR})(\mathrm{X}) \quad$ There exists $\mathbb{Q}^{X} \sim \mathbb{P}$ such that the price process $X$ is an $\left(\mathbb{F}^{X}, \mathbb{Q}^{X}\right)$-local martingale (in other words, $\mathbb{Q}^{X}$ is a local martingale measure for $X$ in its own filtration).

This assumption is introduced for a finer understanding of how the $\mathbb{G}$ price is formed.

Theorem 3.4. Assume that (NFLVRS) holds. Then, for any traded asset $X$, there exist $J^{X}$ and $w^{X}$ all being in $\mathcal{P}(\mathbb{G})$ and $a(\mathbb{G}, \mathbb{P})$-local martingale $M^{X}$ with $M_{0}^{X}=0$, such that for any $t \leq T$ :

$$
X_{t}=X_{0}+J_{t}^{X}+\int_{0}^{t} w_{u}^{X} d\left\langle M^{X}\right\rangle_{u}+M_{t}^{X} .
$$

The process $J^{X}$ satisfies $J_{0}^{X}=0$, is nonincreasing and $d J^{X}$ is singular with respect to $d\left\langle M^{X}\right\rangle$. If $(N F L V R)(X)$ holds, we have the following additional properties:

(a) If $X$ is $\mathbb{F}^{X}$-predictable, the process $J^{X}$ is null.

(b) If $J^{X}$ is not null, then there exist purely discontinuous $\left(\mathbb{F}^{X}, \mathbb{P}\right)$ martingales that are not $(\mathbb{G}, \mathbb{P})$ martingales.

Proof. In view of Remark 3.3, there exists a $(\mathbb{G}, \mathbb{P})$-local martingale $M^{X}$ and a finite variation, $\mathbb{G}$-predictable process $V^{X}$, such that:

$$
X_{t}=X_{0}+V_{t}^{X}+M_{t}^{X}
$$

We can write $V_{t}^{X}=\int_{0}^{t} w_{u}^{X} d\left\langle M^{X}\right\rangle_{u}+J_{t}^{X}$, where $d J^{X}$ is a signed measure that is singular with respect to $d\left\langle M^{X}\right\rangle$ (i.e., the Lebesgue decomposition of $d V^{X}$ with respect to $d\left\langle M^{X}\right\rangle$; see Proposition A.3 in Appendix A.

To show that $J^{X}$ is a nonincreasing process, we use Girsanov's theorem and Theorem A.1 in Appendix A More precisely, let $\tilde{\mathbb{P}}$ be an equivalent $\mathbb{G}$-supermartingale measure for $X$. By Girsanov's theorem the decomposition of $X$ is given by: $X=X_{0}+\left(J^{X}+\tilde{D}^{X}\right)+\tilde{M}^{X}$, where $\tilde{M}^{X}$ is a $(\mathbb{G}, \tilde{\mathbb{P}})$-martingale and: 
(i) $d \tilde{D}^{X} \ll d\left\langle M^{X}\right\rangle$. Hence $d \tilde{D}^{X}$ and $d J^{X}$ are singular.

(ii) the process $J^{X}+\tilde{D}^{X}$ is nonincreasing.

The two above points imply that both $J^{X}$ and $\tilde{D}^{X}$ are nonincreasing (Theorem A.1 (b)).

Further properties of the process $J^{X}$ are found by making use of (NFLVR)(X), that is, the existence of an equivalent local martingale measure for $X$ in its own filtration. For simplicity, as we shall have in the remaining of the proof two filtrations filtrations, capital letters (as $M$ ) are for $\mathbb{G}$ martingales, and small letters ( as $m$ ) for $\mathbb{F}^{X}$ martingales.

This property implies the following $\left(\mathbb{F}^{X}, \mathbb{P}\right)$ decomposition of $X$ :

$$
X_{t}=X_{0}+\int_{0}^{t} v_{u}^{X} d\left\langle m^{X}\right\rangle_{u}^{\left(\mathbb{F}^{X}, \mathbb{P}\right)}+m_{t}^{X} .
$$

for $m^{X}$ an $\left(\mathbb{F}^{X}, \mathbb{P}\right)$ local martingale and $v^{X} \in \mathcal{P}\left(\mathbb{F}^{X}\right)$.

Let $m^{X, c}$ (resp. $m^{X, d}$ ) be the continuous (resp. purely discontinuous) $\mathbb{F}^{X}$-local martingale composing $m^{X}$. The process $X$ being a $(\mathbb{G}, \mathbb{P})$ semimartingale, necessarily, $m^{X, c}$ and $m^{X, d}$ are $(\mathbb{G}, \mathbb{P})$ special semimartingales; we write their canonical decompositions as:

$$
\begin{aligned}
& m^{X, c}=M^{X, c}+A^{X} \\
& m^{X, d}=M^{X, d}+B^{X},
\end{aligned}
$$

where $M^{X, c}\left(\operatorname{resp} M^{X, d}\right)$, the martingale part of the $(\mathbb{G}, \mathbb{P})$-semimartingale $m^{X, c}$ (resp. $m^{X, d}$ ) is continuous (resp. purely discontinuous). Summing up (3) and (4) we obtain $M^{X}=M^{X, c}+M^{X, d}$ and $M^{X, c}$ (resp. $M^{X, d}$ ) are the continuous (resp. purely discontinuous) $\mathbb{G}$-local martingales composing $M^{X}$. The $(\mathbb{G}, \mathbb{P})$ decomposition of $X$ writes:

$$
X_{t}=X_{0}+A_{t}^{X}+B_{t}^{X}+\int_{0}^{t} v_{u}^{X} d\left\langle m^{X}\right\rangle_{u}^{\left(\mathbb{F}^{X}, \mathbb{P}\right)}+M_{t}^{X} .
$$

The process $m^{X, c}$ has the same constancy intervals as $\left\langle m^{X, c}\right\rangle^{\left(\mathbb{F}^{X}, \mathbb{P}\right)}$; similarily, $M^{X, c}$ has the same constancy intervals as $\left\langle M^{X, c}\right\rangle$. But $\left\langle m^{X, c}\right\rangle^{\left(\mathbb{F}^{X}, \mathbb{P}\right)}=\left\langle M^{X, c}\right\rangle$ (when a semimartingale is continuous, its predictable bracket is defined independently of the filtration). Therefore, we can write:

$$
\begin{aligned}
m_{t}^{X, c} & =\int_{0}^{t} \mathbb{1}_{\left\{d\left\langle M^{X, c\rangle}>0\right\}\right.} d m_{s}^{X, c}=\int_{0}^{t} \mathbb{1}_{\left\{d\left\langle M^{X, c}\right\rangle>0\right\}} d\left(M_{s}^{X, c}+A_{s}^{X}\right) \\
& =M_{t}^{X, c}+\int_{0}^{t} \mathbb{1}_{\left\{d\left\langle M^{X, c}\right\rangle>0\right\}} d A_{s}^{X},
\end{aligned}
$$

that is (comparing with 3 ) $), A_{t}^{X}=\int_{0}^{t} \mathbb{1}_{\left\{d\left\langle M^{X, c}\right\rangle>0\right\}} d A_{s}^{X}$. This proves that $d A^{X} \ll d\left\langle M^{X, c}\right\rangle$ and therefore:

$$
d A^{X} \ll d\left\langle M^{X}\right\rangle .
$$

It follows that 
(a) If $X$ is $\mathbb{F}^{X}$ - predictable, then $m^{X}$ is (predictable and hence) continuous. Also, $X$ is $\mathbb{G}$-predictable, and $M^{X}$ is continuous. It follows that $m^{X, d}=M^{X, d}=B^{X}=0$, that is $M^{X}=M^{X, c}$. Also, $\left\langle m^{X}\right\rangle^{\left(\mathbb{F}^{X}, \mathbb{P}\right)}=\left\langle M^{X}\right\rangle$. Consequently, $J^{X}=0$.

(b) Let us suppose that $X$ is not $\mathbb{F}^{X}$ - predictable, that is, $m^{X, d} \neq 0$. Comparing the expression in (2) with the one in (5) and using (6) we see that if $J^{X} \neq 0$, then:

- either $B^{X} \neq 0$, that is, $m^{X, d}$ is not a $(\mathbb{G}, \mathbb{P})$ local martingale,

- or $B^{X}=0$, that is $m^{X, d}$ is a $(\mathbb{G}, \mathbb{P})$ local martingale, and in this case we need to have that $d\left\langle m^{X}\right\rangle^{\left(\mathbb{F}^{X}, \mathbb{P}\right)}$ is not absolutely continuous with $d\left\langle M^{X}\right\rangle$. This in turn implies that $\left\langle m^{X, d}\right\rangle \neq\left\langle m^{X, d}\right\rangle^{\left(\mathbb{F}^{X}, \mathbb{P}\right)}$. In this case, $\left(m^{X, d}\right)^{2}-\left\langle m^{X, d}\right\rangle^{\left(\mathbb{F}^{X}, \mathbb{P}\right)}$ is a purely discontinuous $\left(\mathbb{F}^{X}, \mathbb{P}\right)$ martingale that is not a $(\mathbb{G}, \mathbb{P})$ martingale.

Remark 3.5. Let us assume the following semimartingale decomposition of $X$ relative to $\left(\mathbb{F}^{X}, \mathbb{P}\right)$ :

$$
X_{t}=X_{0}+\int_{0}^{t} v_{u}^{X} d\left\langle m^{X}\right\rangle_{u}^{\left(\mathbb{F}^{X}, \mathbb{P}\right)}+m_{t}^{X},
$$

which is implied by $(N F L V R)(X)$, that is the classical structure condition in the filtration $\mathbb{F}^{X}$. By the preceding theorem, under $(N F L V R S)$ the $(\mathbb{G}, \mathbb{P})$ decomposition of $X$ is:

$$
X_{t}=X_{0}+J_{t}^{X}+\int_{0}^{t} w_{u}^{X} d\left\langle M^{X}\right\rangle_{u}+M_{t}^{X} .
$$

Elements for linking the two decompositions are given within the theorem and its proof. We distinguish several cases:

- If $X$ is predictable, then $J^{X}=0$ and $\left\langle m^{X}\right\rangle^{\left(\mathbb{F}^{X}, \mathbb{P}\right)}=\left\langle M^{X}\right\rangle$. We have $M^{X}=m^{X}+$ $\int\left(v^{X}-w^{X}\right) d\left\langle m^{X}\right\rangle^{\left(\mathbb{F}^{X}, \mathbb{P}\right)}$, in particular, when $m^{X}$ is not a $(\mathbb{G}, \mathbb{P})$ local martingale, the processes $w^{X}$ and $v^{X}$ differ.

- If $X$ is not predictable (in particular $m^{X}$ is not continuous), but assuming that $m^{X}$ is also a $(\mathbb{G}, \mathbb{P})$ local martingale, then the case $J^{X} \neq 0$ is not excluded. This can occur only in cases where $\left\langle m^{X}\right\rangle^{\left(\mathbb{F}^{X}, \mathbb{P}\right)} \neq\left\langle M^{X}\right\rangle$ (see [5] for an example).

- Finally, if $X$ is not predictable and $m^{X}$ is not $(\mathbb{G}, \mathbb{P})$ local martingale, then we can have $J^{X} \neq 0$ when $\left\langle m^{X}\right\rangle^{\left(\mathbb{F}^{X}, \mathbb{P}\right)} \neq\left\langle M^{X}\right\rangle$, but also when $d\left\langle m^{X}\right\rangle^{\left(\mathbb{F}^{X}, \mathbb{P}\right)}$ is absolutely continuous with respect to $d\left\langle M^{X}\right\rangle$. The examples below illustrate these cases.

We consider below two examples of converging prices. We emphasise that from Theorem 3.4. (NFLVRS) also implies a decomposition for $Y$ (with obvious notations):

$$
Y_{t}=Y_{0}+J_{t}^{Y}+\int_{0}^{t} w_{u}^{Y} d\left\langle M^{Y}\right\rangle_{u}+M_{t}^{Y} .
$$

Example 3.6. Let $B^{1}$ and $B^{2}$ be two independent $\mathbb{P}$-Brownian motions with respective natural filtrations $\mathbb{F}^{1}$ and $\mathbb{F}^{2}$; consider that $\theta^{1}$ is an $\mathbb{F}^{1}$ stopping time and $\theta^{2}$ is an $\mathbb{F}^{2}$ 
stopping time (hence, they are predictable), both considered to have absolutely continuous cumulative distribution functions denoted $C^{1}$ and $C^{2}$, and satisfying $C^{1}(T)<1, C^{2}(T)<$ 1.

The following payoff is scheduled at a fixed maturity date $T$ :

$$
\xi=\mathbb{1}_{\left\{\theta_{1}>T\right\}}+\mathbb{1}_{\left\{\theta_{2} \leq T\right\}} \text {. }
$$

We consider the following distinct information sets:

$$
\begin{aligned}
& \mathcal{G}_{t}^{1}:=\mathcal{F}_{t}^{2} \vee \sigma\left(t \wedge \theta^{1}\right), \\
& \mathcal{G}_{t}^{2}:=\mathcal{F}_{t}^{1} \vee \sigma\left(t \wedge \theta^{2}\right),
\end{aligned}
$$

and we assume that the corresponding prices are $X_{t}=\mathbb{E}\left(\xi \mid \mathcal{G}_{t}^{1}\right)$ and $Y_{t}=\mathbb{E}\left(\xi \mid \mathcal{G}_{t}^{2}\right)$.

We have the following $\mathbb{G}^{1}$ martingales, $t \leq T$ :

$$
\mathbb{P}\left(\theta^{1}>T \mid \mathcal{G}_{t}^{1}\right)=\mathbb{1}_{\left\{\theta^{1}>t\right\}} \frac{\mathbb{P}\left(\theta^{1}>T\right)}{\mathbb{P}\left(\theta^{1}>t\right)}=\mathbb{1}_{\left\{\theta^{1}>t\right\}} \frac{1-C^{1}(T)}{1-C^{1}(t)}
$$

(see Proposition 1 in [11]), and:

$$
\mathbb{P}\left(\theta^{2} \leq T \mid \mathcal{G}_{t}^{1}\right)=\mathbb{P}\left(\theta^{2} \leq T \mid \mathcal{F}_{t}^{2}\right),
$$

(as $\theta^{2}$ is independent from $\theta^{1}$ ) i.e., the last process is a Brownian martingale. We deduce that the $\mathbb{G}^{1}$ adapted price for the claim $\xi$ decomposes as follows:

$$
X_{t}=X_{0}-\int_{0}^{t} \frac{1-C^{1}(T)}{1-C^{1}(s)} d \mathbb{1}_{\left\{\theta_{1} \leq s\right\}}+\int_{0}^{t \wedge \theta^{1}}\left(1-C^{1}(T)\right) d\left(1-C^{1}(s)\right)^{-1}+M_{t}^{X},
$$

where $M^{X}=\mathbb{P}\left(\theta^{2} \leq T \mid \mathcal{F}^{2}\right)-\mathbb{P}\left(\theta^{2} \leq T\right)$.

Similar arguments lead to the following $\mathbb{G}^{2}$ adapted price:

$$
Y_{t}=Y_{0}+\int_{0}^{t} \frac{1-C^{2}(T)}{1-C^{2}(s)} d \mathbb{1}_{\left\{\theta_{2} \leq s\right\}}-\int_{0}^{t \wedge \theta^{2}}\left(1-C^{2}(T)\right) d\left(1-C^{2}(s)\right)^{-1}+M_{t}^{Y}
$$

with $M^{Y}=\mathbb{P}\left(\theta^{1}>T \mid \mathcal{F}^{1}\right)-\mathbb{P}\left(\theta^{1}>T\right)$.

One can check that: $\mathbb{F}^{X}=\mathbb{G}^{1}, \mathbb{F}^{Y}=\mathbb{G}^{2}$, while the insider filtration is $\mathbb{G}=\mathbb{F}^{1} \vee \mathbb{F}^{2}$ (i.e., the natural filtration of $\left(B^{1}, B^{2}\right)$ ). The processes $M^{X}$ and $M^{Y}$ are also $\mathbb{G}$ martingales. They are Brownian martingales, from the discussion above. Therefore $\left\langle M^{X}\right\rangle$ and $\left\langle M^{Y}\right\rangle$ are absolutely continuous with respect to the Lebesgue measure. We deduce that $X$ decomposes as in (2) and $Y$ as in (7), with the processes

$$
\begin{aligned}
J^{X}: & =-\int_{0}^{\cdot} \frac{1-C^{1}(T)}{1-C^{1}(s)} d \mathbb{1}_{\left\{\theta_{1} \leq s\right\}} \\
J^{Y}: & =\int_{0}^{\cdot} \frac{1-C^{2}(T)}{1-C^{2}(s)} d \mathbb{1}_{\left\{\theta_{2} \leq s\right\}}
\end{aligned}
$$

being $\mathbb{G}$-predictable. Because $J^{Y}$ is an nondecreasing process, we conclude by Theorem 3.4 that the price process $Y$ does not respect (NFLVRS) for the insider. 
Example 3.7. Let us consider the hitting time by a Brownian motion $B$ of a positive random variable $D$ independent from the Brownian motion:

$$
T^{D}=\inf \left\{t \geq 0 \mid B_{t} \geq D\right\} .
$$

In the filtration $\mathbb{F}=\left(\mathcal{F}_{t}\right)$ given by $\mathcal{F}_{t}:=\sigma\left(T^{D} \wedge s, s \leq t\right)$ we have that $T^{D}$ is a totally inaccessible $\mathbb{F}$-stopping time with corresponding $\mathbb{F}$-intensity process:

$$
c(t)=\frac{\mathbb{1}_{\left\{T^{D}>t\right\}}}{\mathbb{P}\left(T^{D}>t\right)} \int_{0}^{\infty} f_{x}(t) d F_{D}(x),
$$

where $F_{D}(x)$ is the distribution function of $D$ and $f_{x}(t)$ is the density function of the hitting time $T^{x}$. We denote $H_{t}:=\mathbb{1}_{\left\{T^{D} \leq t\right\}}-\int_{0}^{t} c(s) d s$ which is an $\mathbb{F}$-martingale.

Let us assume that the price process $X$ is given by the positive local martingale $X=$ $X_{0} \mathcal{E}(-H)$, that is, it satisfies:

$$
X_{t}=X_{0}-\int_{0}^{t} X_{s_{-}} d H_{s}
$$

One can notice that $\mathbb{F}^{X}=\mathbb{F}$. For simplicity we do not introduce the second asset $Y$ and we rather concentrate on the dynamics of $X$ in the larger filtration $\mathbb{G}$ given by $\mathcal{G}_{t}:=$ $\mathcal{F}_{t}^{X} \vee \sigma\left(B_{s}, s \leq t\right)$.

We denote $\Lambda^{\mathbb{G}}$ the $\mathbb{G}$-compensator of $T^{D}$, so that the process: $H_{t}^{\mathbb{G}}:=\mathbb{1}_{\left\{T^{D} \leq t\right\}}-\Lambda_{t}^{\mathbb{G}}$ is a $\mathbb{G}$-martingale. It can be shown (using [11] and the fact that $\sigma\left(B_{s}, s \leq t\right)$ is immersed in $\mathbb{G})$, that $\Lambda^{\mathbb{G}}$ is absolutely continuous with respect to the measure generated by the running supremum of the Brownian motion:

$$
\Lambda_{t}^{\mathbb{G}}=\int_{0}^{t \wedge T^{D}} \frac{d \mathbb{P}\left(T^{D}>s \mid \mathcal{F}_{s}^{B}\right)}{\mathbb{P}\left(T^{D}>s \mid \mathcal{F}_{s}^{B}\right)}=-\int_{0}^{t \wedge T^{D}} \frac{d F_{D}\left(S_{s}\right)}{1-F_{D}\left(S_{s}\right)}=-\ln \left(1-F_{D}\left(S_{t \wedge T^{D}}\right)\right),
$$

where $\mathbb{F}^{B}$ is the Brownian filtration and $S$ is the running supremum of $B$. The $\mathbb{G}$ decomposition of $X$ writes, using that $H=H^{\mathbb{G}}-\int c(s) d s+\Lambda^{\mathbb{G}}$ :

$$
X_{t}=X_{0}+\left(\int_{0}^{t} X_{s} c(s) d s-\int_{0}^{t} X_{s} d \Lambda_{s}^{\mathbb{G}}\right)-\int_{0}^{t} X_{s-} d H_{s}^{\mathbb{G}}
$$

Using Theorem 3.4 we identify $M_{t}^{X}=-\int_{0}^{t} X_{s-} d H_{s}^{\mathbb{G}}$. From (8) it can be seen that $d\left\langle M^{X}\right\rangle$ is absolutely continuous with respect to $d S$. Therefore, $J_{t}^{X}=\int_{0}^{t} X_{s} c(s) d s$, as the Lebesgue measure is orthogonal with respect to the $d S$. Because $J^{X}$ is nondecreasing, from Theorem 3.4 we conclude that there are arbitrage opportunities, in the sense that (NFLVRS) fails. An arbitrage strategy is easy to implement by the $\mathbb{G}$-informed investor: buy the asset $X$ at any time before $T^{D}$ when the Brownian motion is strictly below its running maximum and sell it any time before it reaches its maximum level again. On these intervals, the price process $X$ is strictly increasing; the arbitrage strategy described performs a strictly positive profit proportional to the holding period of the asset $X$. 


\section{A Result on the Existence of a Supermartingale Measure}

In this section we investigate the existence of a specific $\mathbb{G}$-supermartingale measure for two price processes $X$ and $Y$, that we shall call fundamental supermartingale measure for $(X, Y)$. This object will play an important role, as systematic arbitrage opportunities occur when this supermartingale measure cannot be constructed.

The analysis gains a lot in transparency if we start by assuming the existence of some supermartingale measure for one of the assets, that we shall still call $\mathbb{P}$ for simplicity. More precisely, in this section, we work with the filtered probability space $(\Omega, \mathcal{G}, \mathbb{G}, \mathbb{P})$, where the two assets are assumed to have the following representations:

$$
\begin{aligned}
& X=X_{0}+J^{X}+M^{X}, \\
& Y=Y_{0}+V^{Y}+M^{Y},
\end{aligned}
$$

with $M^{X}$ and $M^{Y}$ being $(\mathbb{G}, \mathbb{P})$-local martingales that are locally square integrable with $M_{0}^{X}=M_{0}^{Y}=0$ and such that the process $V^{Y}$ is a finite variation, $\mathbb{G}$-predictable process. The process $J^{X}$ is considered to be nonincreasing and the measure $d J^{X}$ is orthogonal to $d\left\langle M^{X}\right\rangle$.

Remark 4.1. The decomposition in (9) differs from (2). The existence of such a supermartingale measure $\mathbb{P}$ for $X$-that here is assumed-is a first step to the construction of the fundamental supermartingale measure for the couple $(X, Y)$.

We decompose the martingale $M^{Y}$ as:

$$
M^{Y}=M^{1}+M^{2}
$$

with $M^{1} \in \mathcal{S}\left(M^{X}\right)$ and $M^{2} \in \mathcal{S}\left(M^{X}\right)^{\perp}$ (see Notation 3.1) so that we can write $M^{1}$ as:

$$
M_{t}^{1}=\int_{0}^{t} h_{u} d M_{u}^{X}
$$

for some process $h \in \mathcal{P}(\mathbb{G})$ and assumed to have right-continuous sample path.

We shall need the following additional decompositions:

- The predictable, finite variation part of $Y$ stated in (10) decomposes uniquely as:

$$
V^{Y}=A-a
$$

where $A$ and $a$ are nondecreasing processes which do not increase on the same sets (that is, $d A$ and $d a$ are orthogonal measures) ${ }^{1}$, and $a_{0}=A_{0}=0$.

\footnotetext{
${ }^{1}$ In order to preserve the compatibility with the decomposition result in Theorem 3.4, $d A$ is assumed absolutely continuous with respect to $d\left\langle M^{Y}\right\rangle$. This property will solely be used for constructing an arbitrage portfolio in Lemma 4.7
} 
- The process $A$ in (12) decomposes uniquely as a sum of two other nondecreasing processes:

$$
A=A^{1}+A^{2}
$$

where $d A^{1} \ll h^{+} d\left\langle M^{X}\right\rangle$ and $d A^{2} \perp h^{+} d\left\langle M^{X}\right\rangle$. Therefore, there exist $a^{1} \geq 0$ such that

$$
A_{t}^{1}=\int_{0}^{t} a_{u}^{1} h_{u}^{+} d\left\langle M^{X}\right\rangle_{u}
$$

(the non negativity of $a^{1}$ comes from the fact that $A^{1}$ is nondecreasing ) and we introduce $\tilde{a}^{1}$ so that $\tilde{a}^{1}=\tilde{a}^{1} \mathbb{1}_{\{h>0\}}=\frac{a^{1}}{h} \mathbb{1}_{\{h>0\}} \geq 0$ and

$$
A_{t}^{1}=\int_{0}^{t} \tilde{a}_{u}^{1} d\left\langle M^{1}\right\rangle_{u}=\int_{0}^{t} \tilde{a}_{u}^{1}\left(h_{u}\right)^{2} d\left\langle M^{X}\right\rangle_{u}
$$

We now state our main result of this section:

Theorem 4.2. Assume that $\tilde{a}^{1} \Delta M^{1}<1$ holds almost surely. We consider the following conditions:

(C1) $d A^{2} \ll d\left\langle M^{2}\right\rangle$. We denote $\tilde{a}^{2}$ the density of $d A^{2}$ with respect to $d\left\langle M^{2}\right\rangle$.

(C2) $\tilde{a}^{2} \Delta M^{2}<1$.

(C3) $\mathbb{E}\left[D_{T}^{*}\right]=1$, where:

$$
D_{t}^{*}:=\mathcal{E}_{t}\left(-\int_{0} \tilde{a}_{s}^{1} d M_{s}^{1}\right) \mathcal{E}_{t}\left(-\int_{0}^{\cdot} \tilde{a}_{s}^{2} d M_{s}^{2}\right), \quad t \in[0, T] .
$$

If (C1)-(C3) are satisfied, the price processes $(X, Y)$ satisfy (NFLVRS). Additionally, the probability measure $\mathbb{P}^{*}$ defined as:

$$
\left.\frac{d \mathbb{P}^{*}}{d \mathbb{P}}\right|_{\mathcal{G}_{t}}:=D_{t}^{*}, \quad t \in[0, T]
$$

is a supermartingale measure for $(X, Y)$ that we call the fundamental supermartingale measure for $(X, Y)$.

Conversely, if the price processes $(X, Y)$ satisfy (NFLVRS), then $(C 1)$ and $(C 2)$ hold true, so that the process $D^{*}$ is a strictly positive local martingale.

Remark 4.3. We notice that the probability $\mathbb{P}^{*}$ depends on the initial probability $\mathbb{P}$. This calls for some clarifications about naming $\mathbb{P}^{*}$ fundamental supermartingale measure, especially because $\mathbb{P}$ plays already a particular role (see Remark 4.1). Intuitively, some absolute continuity relations that proved to be crucial in its construction are preserved under an equivalent change of the probability measure. For this reason, there is something systematic (i.e., not depending on $\mathbb{P})$ about the conditions $(C 1)$ and $(C 2)$ : when any of them fails to be true then also (NFLVRS) fails, and obviously, (NFLVRS) does not depend on the probability $\mathbb{P}$ except for fixing the null sets. This is the reason for using the term "fundamental". The following properties are providing some additional insight: 
- Let us write $V^{Y}=J^{Y}+\int w^{Y} d\left\langle M^{Y}\right\rangle$ and assume that $\mathcal{E}\left(-\int w^{Y} d M^{Y}\right)$ is a uniformly integrable martingale. We define $\mathbb{Q}$ via $\left.\frac{d \mathbb{Q}}{d \mathbb{P}}\right|_{\mathcal{G}_{T}}:=\mathcal{E}_{T}\left(-\int w^{Y} d M^{Y}\right)$ Then, under $\mathbb{Q}$, we have the following decompositions (with obvious notations):

$$
\begin{aligned}
& X=X_{0}+V^{X, \mathbb{Q}}+M^{X, \mathbb{Q}}, \\
& Y=Y_{0}+J^{Y}+M^{Y, \mathbb{Q}}
\end{aligned}
$$

which are symmetrical to (9)-(10) under $\mathbb{P}$. A further (and, we have to admit, tedious) analysis consisting of applying Theorem 4.2 under $\mathbb{Q}$ and with the roles of $X$ and $Y$ reversed, leads to the same supermartingale measure $\mathbb{P}^{*}$ as obtained when starting from $\mathbb{P}$.

- Nevertheless, there is no uniqueness of $\mathbb{P}^{*}$. If we consider a change of measure $\mathbb{Q}$ satisfying: the martingale $\mathbb{E}\left(\frac{d \mathbb{Q}}{d \mathbb{P}} \mid \mathcal{G}_{t}\right)$ is orthogonal to $M^{X}$, then, under $\mathbb{Q}$ we have the following decompositions:

$$
\begin{aligned}
& X=X_{0}+J^{X}+M^{X}, \\
& Y=Y_{0}+V^{Y, \mathbb{Q}}+M^{Y, \mathbb{Q}} .
\end{aligned}
$$

Theorem 4.2 can be applied under $\mathbb{Q}$, but we do not obtain in general the same fundamental supermartingale measure as when starting from $\mathbb{P}$.

Before proving the theorem, let us give some simple examples in order to illustrate the various processes involved, in particular the different decompositions of the process $V^{Y}$. Note that we do not consider below that $X$ and $Y$ are converging prices; examples with converging prices are provided in Section 5 .

Example 4.4. Suppose that $B^{1}$ and $B^{2}$ are two independent Brownian motions and

$$
\begin{aligned}
& X_{t}=X_{0}+B_{t \wedge \theta}^{1} \quad \text { with } \theta:=\inf \left\{t \in[0, T], B_{t}=-X_{0}\right\}, T \text { fixed } \\
& Y_{t}=Y_{0}+\int_{0}^{t} F_{s} d s+\int_{0}^{t} H_{s} d B_{s}^{1}+\int_{0}^{t} G_{s} d B_{s}^{2}, t \in[0, T]
\end{aligned}
$$

with $F, G$ and $H$ being predictable processes, that for simplicity we assume bounded. Let us identify the key processes introduced previously in this section.

We have $M_{t}^{X}=B_{t \wedge \theta}^{1}, M_{t}^{1}=\int_{0}^{t \wedge \theta} H_{s} d B_{s}^{1}=\int_{0}^{t} H_{s} d M_{s}^{X}, M_{t}^{2}=\int_{t \wedge \theta}^{t} H_{s} d B_{s}^{1}+\int_{0}^{t} G_{s} d B_{s}^{2}$. Therefore:

$$
\begin{aligned}
\left\langle M^{1}\right\rangle_{t} & =\int_{0}^{t \wedge \theta}\left(H_{s}\right)^{2} d s=\int_{0}^{t}\left(H_{s}\right)^{2} d\left\langle M^{X}\right\rangle_{s} \\
\left\langle M^{2}\right\rangle_{t} & =\int_{0}^{t}\left[\left(H_{s}\right)^{2} \mathbb{1}_{\{\theta \leq s\}}+\left(G_{s}\right)^{2}\right] d s .
\end{aligned}
$$


Moreover, the process $A_{t}=\int_{0}^{t}\left(F_{s}\right)^{+} d s$ decomposes as $A=A^{1}+A^{2}$ with:

$$
\begin{aligned}
& A_{t}^{1}=\int_{0}^{t \wedge \theta} \mathbb{1}_{\left\{H_{s}>0\right\}}\left(F_{s}\right)^{+} d s=\int_{0}^{t} \tilde{a}_{s}^{1} d\left\langle M^{1}\right\rangle_{s}, \quad \text { where } \tilde{a}_{t}^{1}=\frac{\mathbb{1}_{\left\{H_{t}>0\right\}}\left(F_{t}\right)^{+}}{\left(H_{t}\right)^{2}} \\
& A_{t}^{2}=\int_{0}^{t} \mathbb{1}_{\left\{H_{s} \leq 0\right\} \cup\{\theta \leq s\}}\left(F_{s}\right)^{+} d s .
\end{aligned}
$$

The existence of the density process $\tilde{a}^{2}$ is not guaranteed. The absolute continuity condition $(C 1)$ in the Theorem 4.2 becomes: The process $G$ is non null on the set:

$$
\left\{(t, \omega) \mid \theta(\omega)>t, H_{t}(\omega) \leq 0, F_{t}(\omega)>0\right\} \cup\left\{(t, \omega) \mid \theta(\omega) \leq t, H_{t}(\omega)=0, F_{t}(\omega)>0\right\} .
$$

When this is the case, we have $A_{t}^{2}=\int_{0}^{t} \tilde{a}_{s}^{2} d\left\langle M^{2}\right\rangle_{s}$ with

$$
\tilde{a}_{t}^{2}=\mathbb{1}_{\{\theta>t\}} \frac{\mathbb{1}_{\left\{H_{t} \leq 0\right\}}\left(F_{t}\right)^{+}}{\left(G_{t}\right)^{2}}+\mathbb{1}_{\{\theta \leq t\}} \frac{\left(F_{t}\right)^{+}}{\left(H_{t}\right)^{2}+\left(G_{t}\right)^{2}}
$$

and the following process

$$
D^{*}:=\mathcal{E}\left(-\int_{0}^{\theta \wedge}\left(F_{s}\right)^{+}\left(\frac{\mathbb{1}_{\left\{H_{s}>0\right\}}}{H_{s}} d B_{s}^{1}+\frac{\mathbb{1}_{\left\{H_{s} \leq 0\right\}}}{G_{s}} d B_{s}^{2}\right)-\int_{\cdot \wedge \theta}^{\cdot}\left(F_{s}\right)^{+} \frac{H_{s} d B_{s}^{1}+G_{s} d B_{s}^{2}}{\left(H_{s}\right)^{2}+\left(G_{s}\right)^{2}}\right)
$$

is the candidate for the density of the fundamental supermartingale measure. The theorem then states that there exists a super-martingale measure if (the other conditions being fulfilled) $\mathbb{E}\left[D_{T}^{*}\right]=1$.

Example 4.5. Another simple example is the one where $M^{2} \equiv 0$. In this case the theorem simply says that A should not increase on the sets where $d\langle X, Y\rangle<0$, otherwise (NFLVRS) does not hold. See also Subsection 5.1

Example 4.6. If the process $\langle X, Y\rangle$ is strictly increasing, then $A^{2} \equiv 0$ and only the condition $(C 3)$ in the theorem: $\mathbb{E}\left[\mathcal{E}_{T}\left(-\int_{0}^{*} \tilde{a}_{s}^{1} d M_{s}^{1}\right)\right]=1$ needs to be checked. However, if this not fulfilled, we cannot in general conclude to absence of (NFLVRS) as (C3) is not a necessary condition. Consider for instance $X=\mathcal{E}\left(B^{1}\right)$ and $Y=\mathcal{E}\left(\int_{0}^{\cdot} \frac{d s}{\sqrt{T-s}}+B^{1}+\int_{0}^{\cdot} \frac{d B_{s}^{2}}{\sqrt{T-s}}\right)$, again with $B^{1}$ and $B^{2}$ being independent Brownian motions. The density process $D^{*}$ is given by $D^{*}=\mathcal{E}\left(-\int_{0}^{\cdot} \frac{d B_{s}^{1}}{\sqrt{T-s}}\right), D_{T}^{*}=0$ and is a strict local martingale (see Example 2.2 in [16]), hence the fundamental supermartingale measure is not well defined. However $(N F L V R)$ holds, as the probability $\mathbb{Q}$ is $\frac{d \mathbb{Q}}{d \mathbb{P}}=\mathcal{E}_{T}\left(-B^{2}\right)$ is an equivalent local martingale measure for $(X, Y)$.

Proof. (Proof of the Theorem 4.2)

$" \Rightarrow$ " Condition (C1) ensures the existence of a process $\tilde{a}^{2}$, such that:

$$
A^{2}=\int_{0}^{\cdot} \tilde{a}_{u}^{2} d\left\langle M^{2}\right\rangle_{u}
$$


and $\tilde{a}^{2}$ is nonnegative, due to the nondecreasing property of $A^{2}$. Condition (C3), implies that the process $D$ is a martingale, while condition $(\mathrm{C} 2)$ together with the condition $\tilde{a}^{1} \Delta M^{1}<1$ ensure that it is strictly positive.

We define:

$$
\left.\frac{d \mathbb{P}^{*}}{d \mathbb{P}}\right|_{\mathcal{G}_{T}}:=D_{T}^{*}
$$

which is indeed an equivalent probability measure. It is easy to check that it is a supermartingale measure: indeed, under $\mathbb{P}^{*}$,

$$
d X_{t}=d J_{t}^{X}-\tilde{a}_{t}^{1} h_{t} d\left\langle M^{X}\right\rangle_{t}+d M_{t}^{X, *}=d J_{t}^{X}-\tilde{a}_{t}^{1}\left(h_{t}\right)^{+} d\left\langle M^{X}\right\rangle_{t}+d M_{t}^{X, *}
$$

where $M^{X, *}$ is a $\left(\mathbb{G}, \mathbb{P}^{*}\right)$-local martingale. The processes $J^{X}$ and $-\int \tilde{a}_{s}^{1}(h)_{s}^{+} d\left\langle M^{X}\right\rangle_{s}$ being nonincreasing, $X$ is a supermartingale under $\mathbb{P}^{*}$. Also:

$d Y_{t}=d V_{t}^{Y}+d M_{t}^{Y}=d A_{t}^{1}+d A_{t}^{2}-d a_{t}+d M_{t}^{Y, *}-\tilde{a}_{t}^{1}\left(h_{t}\right)^{2} d\left\langle M^{X}\right\rangle_{t}-\tilde{a}_{t}^{2} d\left\langle M^{2}\right\rangle_{t}=d M_{t}^{Y, *}-d a_{t}$ where $M^{Y, *}$ is a $\left(\mathbb{G}, \mathbb{P}^{*}\right)$-local martingale.

$" \Leftarrow "$ We assume that there exists an equivalent supermartingale measure, that we denote $\widetilde{\mathbb{P}}$. Without loss of generality, the density process has the representation

$$
\left.\frac{d \widetilde{\mathbb{P}}}{d \mathbb{P}}\right|_{\mathcal{G}_{t}}=\mathcal{E}_{t}(-L)
$$

where $L$ can be decomposed as:

$$
L_{t}=\int_{0}^{t} \ell_{u}^{1} d M_{u}^{1}+\int_{0}^{t} \ell_{u}^{2} d M_{u}^{2}+U_{t} .
$$

with $U$ a local martingale orthogonal to both $M^{1}$ and $M^{2}$.

The processes $X$ and $Y$ are $\widetilde{\mathbb{P}}$-supermartingales; therefore we need to have simultaneously:

(i) $\left(\left\langle M^{X}, L\right\rangle_{t}, t \in[0, T]\right)$ is an nondecreasing process;

(ii) $\left(\int_{0}^{t} \mathbb{1}_{\{d A \neq 0\}} d\left\langle M^{Y}, L\right\rangle_{u}-A_{t}, t \in[0, T]\right)$ is an nondecreasing process.

Condition (i) is obtained as follows. The process $X$ is a $\widetilde{\mathbb{P}}$-supermartingale if and only if $J^{X}-\left\langle M^{X}, L\right\rangle$ is a nonincreasing process. But $J^{X}$ is nonincreasing and $d J^{X}$ is singular to $d\left\langle M^{X}\right\rangle$, therefore the condition (i) appears as necessary and sufficient for $X$ to be a $\widetilde{\mathbb{P}}$-supermartingale.

Also, some clarifications concerning the condition (ii) above. The process $Y$ is a $\widetilde{\mathbb{P}}$ supermartingale if and only if $V^{Y}-\left\langle M^{Y}, L\right\rangle$ is a nonincreasing process. But $V^{Y}-\left\langle M^{Y}, L\right\rangle$ is nonincreasing if and only if the two processes $\left(A_{t}-\int_{0}^{t} \mathbb{1}_{\{d A \neq 0\}} d\left\langle M^{Y}, L\right\rangle_{u}, t \in[0, T]\right)$ and $\left(-a_{t}-\int_{0}^{t} \mathbb{1}_{\{d a \neq 0\}} d\left\langle M^{Y}, L\right\rangle_{u}, t \in[0, T]\right)$ are nonincreasing. However, the last condition is not going to be exploited here. 
From condition (i) above, we obtain that necessarily the process $h \ell^{1}$ is nonegative. In particular on the set $\{h<0\}$ the process $\ell^{1}$ has negative or null values only:

$$
\ell^{1} \mathbb{1}_{\{h<0\}} \leq 0 .
$$

Let us now analyze condition (ii). For simplicity, we denote: $\tilde{\ell}_{t}^{1}:=\ell_{t}^{1} \mathbb{1}_{\{d A \neq 0\}}$ and $\tilde{\ell}_{t}^{2}:=$ $\ell_{t}^{2} \mathbb{1}_{\{d A \neq 0\}}$. From $(\sqrt{16})$, the process $\tilde{\ell}^{1}$ satisfies as well:

$$
\tilde{\ell}^{1} \mathbb{1}_{\{h<0\}} \leq 0,
$$

We recall that the process $A$ decomposes as $\int_{0}^{t} \tilde{a}_{u}^{1} d\left\langle M^{1}\right\rangle_{u}+A_{t}^{2}$, with $\tilde{a}^{1}$ satisfying $\tilde{a}^{1}=$ $\tilde{a}^{1} \mathbb{1}_{\{h>0\}}$ and hence:

$$
\begin{aligned}
& \int_{0}^{t} \mathbb{1}_{\{d A \neq 0\}} d\left\langle M^{Y}, L\right\rangle_{u}-A_{t}= \\
& \quad=\int_{0}^{t}\left(\tilde{\ell}_{u}^{1}-\tilde{a}_{u}^{1}\right) \mathbb{1}_{\left\{h_{u}>0\right\}} d\left\langle M^{1}\right\rangle_{u}+\int_{0}^{t} \tilde{\ell}_{u}^{2} d\left\langle M^{2}\right\rangle_{u}-\left(A_{t}^{2}-\int_{0}^{t} \tilde{\ell}_{u}^{1} \mathbb{1}_{\left\{h_{u} \leq 0\right\}} d\left\langle M^{1}\right\rangle_{u}\right) .
\end{aligned}
$$

The process above should be nondecreasing . Because both processes $A^{2}$ and $-\int_{0}^{\cdot} \tilde{\ell}_{u}^{1} \mathbb{1}_{\left\{h_{u} \leq 0\right\}} d\left\langle M^{1}\right\rangle_{u}=$ $-\int_{0}^{\cdot} \tilde{\ell}_{u}^{1} \mathbb{1}_{\left\{h_{u}<0\right\}} d\left\langle M^{1}\right\rangle_{u}$ are nondecreasing (see 17$\left.\}\right)$ and they do not increase (i.e., they stay constant) on the set $\left\{h_{t}>0\right\}$, it follows that the process:

$$
\int_{0}^{\cdot} \mathbb{1}_{\left\{h_{u} \leq 0\right\}} \tilde{\ell}_{u}^{2} d\left\langle M^{2}\right\rangle_{u}-C
$$

needs to be nondecreasing, where $C_{t}:=A_{t}^{2}-\int_{0}^{t} \tilde{\ell}_{u}^{1} \mathbb{1}_{\left\{h_{u} \leq 0\right\}} d\left\langle M^{1}\right\rangle_{u}$ is nondecreasing . It follows from Theorem A.1 in the Appendix A that $C$ is absolutely continuous with respect to $d\left\langle M^{2}\right\rangle$. Because $C$ is the sum of two nondecreasing processes, then each term should be absolutely continuous with respect to $d\left\langle M^{2}\right\rangle$, that is:

$$
\int_{0}^{t} \tilde{\ell}_{u}^{1} \mathbb{1}_{\left\{h_{u} \leq 0\right\}} d\left\langle M^{1}\right\rangle_{u}=\int_{0}^{t} \tilde{\ell}_{u}^{1} \mathbb{1}_{\left\{h_{u} \leq 0\right\}} e_{u} d\left\langle M^{2}\right\rangle_{u}
$$

for some nonnegative process $\left(e_{t}\right)$, and

$$
A_{t}^{2}=\int_{0}^{t} \tilde{a}_{u}^{2} d\left\langle M^{2}\right\rangle_{u}
$$

for a nonnegative process $\tilde{a}^{2}=\tilde{a}^{2} \mathbb{1}_{\{h \leq 0\}}$. It follows that the condition (C1) in the theorem must hold. In particular, the local martingale $D^{*}$ exists.

It remains to show that $(\mathrm{C} 2)$ holds as well, a property that triggers the strict positivity of the local martingale $D^{*}$. Below we show that $(\mathrm{C} 2)$ is a consequence of the strict positivity of the local martingale $\mathcal{E}(-L)$ in $(15)$. We notice first that, the process in 18 being nondecreasing :

$$
\left(\tilde{a}^{2}-\tilde{\ell}^{1} e-\tilde{\ell}^{2}\right) \mathbb{1}_{\{h \leq 0\}} \leq 0
$$


and therefore:

$$
0 \leq \tilde{a}^{2} \leq\left(\tilde{\ell}^{1} e+\tilde{\ell}^{2}\right) \mathbb{1}_{\{h \leq 0\}} \leq \tilde{\ell}^{2} \mathbb{1}_{\{h \leq 0\}} .
$$

To obtain the last inequality above, we use $\tilde{\ell}^{1} e \mathbb{1}_{\{h \leq 0\}} \leq 0$ (e being a positive process). Indeed: $\mathbb{1}_{\{h<0\}} \tilde{\ell}^{1} \leq 0$ as in 17$), \mathbb{1}_{\{h=0\}} d\left\langle M^{X}\right\rangle=0$ and therefore, using the equality 19$\}$ the process $\mathbb{1}_{\{h=0\}} \tilde{\ell}^{1} e$ is null.

As the process $\mathcal{E}(-L)$ in $(15)$ is strictly positive, and from the orthogonality of $M^{1}$ and $M^{2}$, it follows that we must have: $-\ell^{1} \Delta M^{1}>-1$ and $-\ell^{2} \Delta M^{2}>-1$. In particular, the last inequality holds on the set $\left\{\tilde{a}^{2}>0\right\} \cap\{h \leq 0\}$ (notice that on this set we have $\ell^{2}>0$, which follows from (20)). Then, the inequalities in (20) ensure that

$$
-\tilde{a}^{2} \Delta M^{2}>-1
$$

Indeed, 20 implies that $-\tilde{a}^{2} \geq-\tilde{\ell}^{2} \mathbb{1}_{\{h \leq 0\}}$, hence, if $\Delta M^{2}>0$, one has $-\tilde{a}^{2} \Delta M^{2} \geq$ $-\tilde{\ell}^{2} \mathbb{1}_{\{h \leq 0\}} \Delta M^{2} \geq-\mathbb{1}_{\{h \leq 0\}} \geq-1$. If $\Delta M^{2}<0$, one has $-\tilde{a}^{2} \Delta M^{2} \geq 0>-1$. Therefore the condition $(\mathrm{C} 2)$ in the theorem holds as well.

Theorem 4.2 emphasizes the fact that the condition (C1) is necessary for (NFLVRS) to hold. In the remaining of this section we reveal a systematic arbitrage portfolio when $(\mathrm{C} 1)$ fails. For this, we identify the set where the condition fails (i.e., the arbitrage set):

$$
\mathcal{A}:=\left\{(\omega, t) \in \Omega \times[0, T(\omega)] \mid d A_{t}^{2}(\omega)>0 \text { and } d\left\langle M^{2}\right\rangle_{t}(\omega)=0\right\} ;
$$

in other words, in $\mathcal{A}$ the measure $d A^{2}$ is not absolutely continuous with respect to $d\left\langle M^{2}\right\rangle$. The condition $(\mathrm{C} 1)$ can be rewritten as: $\mathbb{P}(\omega: \exists t,(\omega, t) \in \mathcal{A})=0$.

We introduce the début of $\mathcal{A}$ :

$$
D_{\mathcal{A}}:=\inf \{t \geq 0 \mid(\omega, t) \in \mathcal{A}\},
$$

with the usual convention: $\inf \emptyset=\infty$.

The random time $D_{\mathcal{A}}$ is a predictable $\mathbb{G}$ stopping time. This can be proved as follows. The processes $A^{2}$ and $\left\langle M^{2}\right\rangle$ are $\mathbb{G}$-predictable, hence the set $\mathcal{A}$ is $\mathbb{G}$-predictable. Furthermore, $A^{2}$ and $\left\langle M^{2}\right\rangle$ are right continuous, so that $\llbracket D_{\mathcal{A}} \rrbracket \subset \mathcal{A}$. We conclude using Proposition 2.40, p. 354 in [23].

The exit time from $\mathcal{A}$ :

$$
E_{\mathcal{A}}:=\inf \left\{t>D_{\mathcal{A}} \mid(\omega, t) \notin \mathcal{A}\right\}
$$

is as well a predictable stopping time (it can be also written as the début of the set $\{(\omega, t) \in$ $\Omega \times \llbracket D_{\mathcal{A}} \wedge T, T \rrbracket \mid d A_{t}^{2}(\omega)=0$ or $\left.\left.d\left\langle M^{2}\right\rangle_{t}(\omega)>0\right\}\right)$.

To construct our arbitrage portfolio we use a trading strategy $\pi=\left(\pi^{C}, \pi^{X}, \pi^{Y}\right)$, where $\pi_{t}^{X} \geq 0$ represents the quantity of asset $X$ in the portfolio at time $t, \pi_{t}^{Y} \geq 0$ the quantity of asset $Y$ and $\pi_{t}^{C} \in \mathbb{R}$ is the amount invested in the risk-free asset (cash) at time $t$ to have a 
self financing strategy (see Definition 2.2). We recall that the value of the portfolio at time $t \in[0, T]$ writes:

$$
V_{t}^{\pi}:=\pi_{t}^{C}+\pi_{t}^{X} X_{t}+\pi_{t}^{Y} Y_{t} .
$$

Additionally, our arbitrage portfolio will satisfy the following conditions:

(a) it is initiated at time $D_{\mathcal{A}}$ at no cost: $V_{D_{\mathcal{A}}}^{\pi}=0$.

(b) at some $\mathbb{G}$ stopping time $S \leq T$ the portfolio has positive value: $V_{S}^{\pi} \geq 0$ a.s. with $\mathbb{P}\left(V_{S}^{\pi}>0\right)>0$. In our case $S$ is any stopping time less or equal to $E_{\mathcal{A}}$.

(c) the underlying trading strategy $\pi$ is admissible in the sense of the Definition 2.2 (some of the admissibility conditions are already implied by the previous points).

Such a portfolio is indeed the following one: $\pi_{0}=(0,0,0)$ (that is, no initial investment), then the self financing strategy associated with

$$
\begin{aligned}
& \pi_{t}^{X}=-h_{t} \mathbb{1}_{\left\{t \in \llbracket D_{\mathcal{A}}, E_{\mathcal{A}} \llbracket\right\}} \\
& \pi_{t}^{Y}=\mathbb{1}_{\left\{t \in \llbracket D_{\mathcal{A}}, E_{\mathcal{A}} \llbracket\right\}} .
\end{aligned}
$$

The lemma below shows that the portfolio value is nondecreasing, in particular it is bounded from below, which ensures that the underlying trading strategies are admissible, that is, (c) is satisfied. It also proves that condition (b) holds (i.e., the portfolio is an arbitrage) as soon as we have a violation of $(\mathrm{C} 1)$, that is: $\mathbb{P}(\omega: \exists t,(\omega, t) \in \mathcal{A})>0$.

Lemma 4.7. The value of a self-financing portfolio $V^{\pi}$ with $\pi$ as in (22)-(23) is an nondecreasing process, and strictly increasing for $(\omega, t) \in \mathcal{A}$.

Proof. The portfolio value is constant outside the set $\mathcal{A}$, therefore we only need to investigate the behaviour of the prices processes $X$ and $Y$ inside the set $\mathcal{A}$.

The portfolio being self-financing, we have:

$$
\begin{aligned}
d V_{t}^{\pi} & =-h_{t} d X_{t}+d Y_{t}=\left(-h_{t} d J_{t}^{X}-h_{t} d M_{t}^{X}\right)+\left(d V_{t}^{Y}+h_{t} d M_{t}^{X}+d M_{t}^{2}\right) \\
& =-h_{t} d J_{t}^{X}+d A_{t}^{2}+d M_{t}^{2} .
\end{aligned}
$$

The last equality appears as a consequence of the fact that in $\mathcal{A}$ we have $d A^{2}>0$ so that $d a=d A^{1}=0$ and $d V^{Y}=d A^{2}$.

But the process $J^{X}$ is necessarily constant in $\mathcal{A}$. This is is because $d J^{X}$ is singular to $d\left\langle M^{X}\right\rangle$ (by definition of $J^{X}$ ), and we do have $d\left\langle M^{X}\right\rangle>0$ for all $(\omega, t) \in \mathcal{A}$. The last property can be seen form the following arguments. We recall the following properties: $d A^{2}$ is absolutely continuous with respect to $d\left\langle M^{Y}\right\rangle=d\left\langle M^{1}\right\rangle+d\left\langle M^{2}\right\rangle$ (consequence of the fact that $d A$ is absolutely continuous with respect to $d\left\langle M^{Y}\right\rangle$, see footnote 1 page 9); and inside $\mathcal{A}$ we have $d A^{2}$ is orthogonal to $d\left\langle M^{2}\right\rangle$. It follows that inside $\mathcal{A}, d A^{2}$ is absolutely continuous with respect to $d\left\langle M^{1}\right\rangle$ and hence also with respect to $d\left\langle M^{X}\right\rangle$. Consequently, $d\left\langle M^{X}\right\rangle>0$ for all $(\omega, t) \in \mathcal{A}$. 
We deduce that the dynamics of the portfolio's value can be rewritten:

$$
d V_{t}^{\pi}=d A_{t}^{2}+d M_{t}^{2} \text { for }(\omega, t) \in \mathcal{A} .
$$

We now notice that inside $\mathcal{A}$ we have $d\left\langle M^{2}\right\rangle \equiv 0$, by definition of $\mathcal{A}$, which implies that $M^{2}$ is constant inside $\mathcal{A}$. This simplifies the dynamics of $V^{\pi}$ :

$$
d V_{t}^{\pi}=d A_{t}^{2} \text { for }(\omega, t) \in \mathcal{A}
$$

that is, $V^{\pi}$ is strictly increasing for $(\omega, t) \in \mathcal{A}$.

\section{Some Examples of CONVERging Prices}

We keep the notation of Section 4 and consider the specific case of converging prices, i.e. $X_{T}=Y_{T}=\xi$ a.s.. Whenever appearing, $\mathbb{Q}^{X}$ (resp. $\mathbb{Q}^{Y}$ ) is an equivalent local martingale measure for $X$ in the filtration $\mathbb{F}^{X}$ (resp. for $Y$ in the filtration $\mathbb{F}^{Y}$ ).

5.1. The martingale $M^{2}$ is null. In this case, we can derive the following quadratic covariation rule:

Lemma 5.1. We suppose that $X$ and $Y$ satisfy the hypotheses from the previous section with $M^{2} \equiv 0$. If (NFLVRS) holds then the process:

$$
\int_{0}^{t} \mathbb{1}_{\{d\langle X, Y\rangle \leq 0\}} d Y_{s}
$$

is a $(\mathbb{G}, \mathbb{P})$-supermartingale, which is to say:

$$
\int_{0}^{t} \mathbb{1}_{\{d\langle X, Y\rangle \leq 0\}} d V_{s}^{Y}
$$

is a nonincreasing process.

Proof. The result follows as an application of the Theorem 4.2. We give an intuitive explanation. The search of supermartingale measures for $X$ and $Y$ requires an analysis of the finite variation parts of $\mathbb{Q}$ decompositions of $X$ and $Y$, for different probability measures $\mathbb{Q}$, with $\mathbb{Q} \sim \mathbb{P}$. Let us denote these $V^{X, \mathbb{Q}}$ and $V^{Y, \mathbb{Q}}$ respectively. A measure $\mathbb{Q}$ such that $V^{X, \mathbb{Q}}$ and $V^{Y, \mathbb{Q}}$ are nonincreasing is a supermartingale measure. On the set where the quadratic covariation process $\langle X, Y\rangle$ is nonincreasing (that is on the set $\{d\langle X, Y\rangle \leq 0\}$ ), a change of measure from $\mathbb{P}$ to a given $\mathbb{Q}$, has opposite effects on $V^{X, \mathbb{Q}}$ and $V^{Y, \mathbb{Q}}$ : when one is decreased, the other increased (as compared to their $\mathbb{P}$ counterparts). We have $V^{X, \mathbb{P}}=J^{X}$ and is constant on $\{d\langle X, Y\rangle \leq 0\}$; suppose that $V^{Y, \mathbb{P}}\left(=V^{Y}\right)$ is (strictly) increasing on $\mathcal{S} \subset\{d\langle X, Y\rangle \leq 0\}$. Then, if there is a probability $\mathbb{Q}$ such that $V^{Y, \mathbb{Q}}$ is nonincreasing in $\mathcal{S}$, we necessarily have $V^{X, \mathbb{Q}}$ nondecreasing in $\mathcal{S}$. So, there is no supermartingale measure for the couple $(X, Y)$ in this case.

We give below some examples of converging assets. 
First, let us suppose $T$ is constant, that $M^{X}$ is a continuous martingale with deterministic quadratic variation, $f$ a deterministic function and $F(t)=\int_{0}^{t} f(s) d\left\langle M^{X}\right\rangle_{s}$. Then, the consider the following prices:

$$
\begin{aligned}
& X_{t}=X_{0}+M_{t}^{X} \\
& Y_{t}=X_{0}-\int_{0}^{t} M_{s}^{X} f(s) d\left\langle M^{X}\right\rangle_{s}+\int_{0}^{t} h_{s} d M_{s}^{X} .
\end{aligned}
$$

with

$$
h_{t}=1+F(T)-F(t) .
$$

An integration by parts shows that $X$ and $Y$ have converging prices, $X_{T}=Y_{T}$ a.s.. Consider for instance $F(t)=1-e^{r t}$ for some $r>0$ (and implicitly $f(t)<0$ ), then the process $h_{t}=1+e^{r t}-e^{r T}$ is negative in an interval of the form $[0, S]$ with $S<T$, provided that $T$ is large enough. By Lemma 5.1, there are arbitrage opportunities if the martingale $M^{X}$ has positive excursions in the interval $[0, S]$.

As a second example, let us analyse the case of Brownian diffusions. Suppose that $B$ is a $(\mathbb{G}, \mathbb{P})$ Brownian motion, $f(t, x)$ and $\sigma(t, x)$ are two Lipschitz continuous and strictly positive functions on $\mathbb{R}_{+} \times \mathbb{R}$, and $x_{0}$ and $y_{0}$ are some positive constants. We consider the following prices

$$
\begin{aligned}
& X_{t}=x_{0}+\int_{0}^{t} \sigma^{X}\left(s, B_{s}\right) d B_{s} \\
& Y_{t}=y_{0}+\int_{0}^{t} f\left(s, B_{s}\right) d s+\int_{0}^{t} \sigma^{Y}\left(s, B_{s}\right) d B_{s}
\end{aligned}
$$

and give conditions under which they are convergent prices. Assume that

$$
\sigma^{Y}(t, x)=\sigma^{X}(t, x)-\frac{\partial g}{\partial x}(t, x)
$$

with $g$ being defined by $g(t, x)=\mathbb{E}\left[\int_{t}^{T} f\left(s, B_{s}\right) d s \mid B_{t}=x\right]$ and set $y_{0}=x_{0}-\mathbb{E}\left[\int_{0}^{T} f\left(s, B_{s}\right) d s\right]$. Then, $X_{T}=Y_{T}$. There are arbitrage opportunities as soon as $d\langle X, Y\rangle \leq 0$, that is:

$$
\sigma^{X}(t, x) \leq \frac{\partial}{\partial x} g(t, x)
$$

Let us now consider the case of a "survival claim": $\xi=\mathbb{1}_{\{\tau>T\}}$, i.e., that pays one monetary unit if some event $\tau$ does not occur before some fixed maturity $T$. Suppose that for all investors $\tau$ is a totally inaccessible stopping time; it admits a constant $\left(\mathbb{F}^{X}, \mathbb{Q}^{X}\right)$ intensity 
$\lambda^{X}$, resp. a constant $\left(\mathbb{F}^{Y}, \mathbb{Q}^{Y}\right)$ intensity $\lambda^{Y}$. In this case, $X$ (resp. $Y$ ) is increasing on the stochastic interval $[0, \tau \wedge T)$ and has a downward jump at $\tau$ if $\tau \leq T$. More precisely:

$$
\begin{aligned}
X_{t} & =\mathbb{Q}^{X}\left(\tau>T \mid \mathcal{F}_{t}^{X}\right)=\mathbb{1}_{\{\tau>t\}} e^{-\lambda^{X}(T-t)} \\
Y_{t} & =\mathbb{Q}^{Y}\left(\tau>T \mid \mathcal{F}_{t}^{Y}\right)=\mathbb{1}_{\{\tau>t\}} e^{-\lambda^{Y}(T-t)} .
\end{aligned}
$$

(NFLVRS) holds in this model (for instance $\mathbb{Q}^{m}$ is supermartingale measure with $m=$ $\arg \max _{i \in\{X, Y\}} \lambda^{i}$ ). This is in line with Lemma 5.1. $[X, Y]_{t}=\Delta X_{\tau} \Delta Y_{\tau} \mathbb{1}_{\{\tau \leq t\}} \geq 0$ and hence $\langle X, Y\rangle \geq 0$.

Now, consider an alternative of the above example, where in the filtration $\mathbb{F}^{X}$, the stopping time $\tau$ is predictable, but it is totally inaccessible in $\mathbb{F}^{Y}$ with constant intensity, i.e., $Y$ is increasing on the stochastic interval $[0, \tau \wedge T)$ and has a downward jump at $\tau$ if $\tau \leq T$ as above. In the filtration $\mathbb{G}$ the stopping time $\tau$ is predictable (because it is predictable in $\mathbb{F}^{X} \subset \mathbb{G}$ ), therefore the price process $Y$ appears to be $\mathbb{G}$-predictable and of finite variation, in particular $\langle X, Y\rangle \equiv 0$. Then, there are arbitrage opportunities: in the filtration $\mathbb{G}$ there is no change of measure to make it a supermartingale. An obvious arbitrage strategy consists in buying $Y$ and selling it just before $\tau$.

5.2. Investors with similar risk attitudes in the two markets. Let us assume that: $X_{t}=$ $\mathbb{E}\left[\xi \mid \mathcal{F}_{t}^{X}\right]$ and $Y_{t}=\mathbb{E}\left[\xi \mid \mathcal{F}_{t}^{Y}\right]$. We interpret this as investors having similar risk attitudes, because $\mathbb{P}$ acts as a martingale measure on both markets individually, where the same payoff $\xi$ is priced in a manner compatible with no arbitrage (more exactly (NFLVR)). In the larger filtration $\mathbb{G}$ nevertheless, there is no guarantee of no arbitrage.

We illustrate with an example of a defaultable asset: $\xi=\mathbb{1}_{\{\tau>T\}} \mathcal{E}(B)_{T}$, the maturity $T$ being fixed. We assume that $B$ is a Brownian motion and $\tau$, the default time of the issuer is an exponentially distributed random variable wih parameter $\lambda$, which is independent from the Brownian motion $B$.

We assume that the following information sets are available for each of the two markets and the insider, respectively, for $t \in[0, T]$ :

$$
\begin{aligned}
\mathcal{F}_{t}^{X} & =\sigma\left(B_{T}\right) \vee \sigma(\tau \wedge s, s \leq t) \\
\mathcal{F}_{t}^{Y} & =\sigma\left(B_{s}, s \leq t\right) \vee \sigma(\tau) \\
\mathcal{G}_{t} & =\sigma\left(B_{s}, s \leq t\right) \vee \sigma\left(B_{T}\right) \vee \sigma(\tau) .
\end{aligned}
$$

We denote

$$
N_{t}:=\mathbb{1}_{\{\tau \leq t\}}-\lambda(t \wedge \tau),
$$

which is an $\mathbb{F}^{X}$-martingale. Also, we notice that the $\mathbb{F}^{Y}$ Brownian motion $B$ is a semimartingale in the larger filtration $\mathbb{G}$, namely

$$
B_{t}=-\int_{0}^{t \wedge T} \frac{B_{T}-B_{u}}{T-u} d u+\beta_{t}
$$

with $\beta$ being a $(\mathbb{G}, \mathbb{P})$ Brownian motion. 
Using $\mathbb{1}_{\{\tau>t\}} e^{\lambda t}=\mathcal{E}_{t}(-N)$, we obtain that:

$$
X_{t}=\mathbb{1}_{\{\tau>t\}} e^{-\lambda(T-t)} \mathcal{E}_{T}(B)=\mathcal{E}_{T}(B) e^{-\lambda T}-\int_{0}^{t} X_{s-} d N_{s},
$$

which is an $\mathbb{F}^{X}$-martingale. However, in the filtration $\mathbb{G}$ the process $X$ is predictable and of finite variation. As it is nondecreasing, we conclude by Theorem 3.4 that $X$ does not fulfil (NFLVRS). On the other hand, $Y$ is given by the following $\mathbb{F}^{Y}$-martingale:

$$
Y_{t}=\mathbb{1}_{\{\tau>T\}} \mathcal{E}(B)_{t}=\mathbb{1}_{\{\tau>T\}}+\int_{0}^{t} Y_{u} d B_{u}
$$

while in the larger filtration $\mathbb{G}$, the following decomposition holds for $Y$ :

$$
Y_{t}=\mathbb{1}_{\{\tau>T\}}-\int_{0}^{t} Y_{u} \frac{B_{T}-B_{u}}{T-u} d u+\int_{0}^{t} Y_{u} d \beta_{u} .
$$

The integral $\int_{0}^{t} Y_{u} \frac{B_{T}-B_{u}}{T-u} d u$ is well defined: indeed, from [18] this condition is equivalent to $\int_{0}^{T} \frac{Y_{s}}{\sqrt{T-s}} d s<\infty$ and, since $E\left(Y_{t}\right) \leq 1$ one has $\mathbb{E}\left(\int_{0}^{T} \frac{\left|Y_{s}\right|}{\sqrt{T-s}} d s\right)<\infty$. This type of model is known for not satisfying (NFLVR). Imposing short sales constraints for the insider does not prevent the free lunches. The candidate density process for the fundamental supermartingale measure is:

$$
D_{t}^{*}=\mathcal{E}_{t}\left(\int_{0}^{\cdot} \frac{\left(B_{T}-B_{u}\right)^{+}}{T-u} d \beta_{u}\right)
$$

the fact that $\frac{\left(B_{T}-B_{u}\right)^{+}}{T-u}$ is not square integrable prevents it from being a valid change of measure. See, e.g., [1, Section 4.2.1].

5.3. Different risk attitudes in the two markets. Here we assume that the two markets have different equivalent martingale measures. We work directly in the filtration $\mathbb{G}$, generated by two independent $\mathbb{P}$-Brownian motions $B$ and $\beta$. We denote $W:=\rho B+\sqrt{1-\rho^{2}} \beta$ for some $\rho \in[-1,1]$. The two asset prices are supposed to be as follows:

$$
\begin{aligned}
X_{t} & =X_{0}+B_{t} \\
Y_{t} & =\mathbb{E}^{\mathbb{Q}^{Y}}\left[X_{T} \mid \mathcal{G}_{t}\right],
\end{aligned}
$$

with

$$
\left.\frac{d \mathbb{Q}^{Y}}{d \mathbb{P}}\right|_{\mathcal{G}_{t}}:=\mathcal{E}\left(-\int_{0}^{\cdot} W_{u}^{Y} d B_{u}\right)_{t},
$$

and with $W^{Y}$ satisfying $d W_{t}^{Y}=\rho W_{t}^{Y} d t+d W_{t}$. We consider $X_{0}>0$ and $T=\inf \{t \geq$ $\left.0, X_{t}=0\right\} \wedge \bar{T}$ with $\bar{T}$ non random (so that the price processes are positive). 
Under the above assumptions, the processes $W^{Y}, B_{t}^{Y}=B_{t}+\int_{0}^{t} W_{u}^{Y} d u$ and $\beta$ are $\mathbb{Q}^{Y}$ Brownian motions. It can be easily computed that $Y$ has the following $(\mathbb{G}, \mathbb{P})$ decomposition:

$$
Y_{t}=X_{0}+\int_{0}^{t}(1-\rho(T-u)) W_{u}^{Y} d u+\int_{0}^{t}(1-\rho(T-u)) d B_{u}-\sqrt{1-\rho^{2}} \int_{0}^{t}(T-u) d \beta_{u} .
$$

We have for $t \leq T$ :

$$
\begin{aligned}
M_{t}^{1} & =\int_{0}^{t} h_{u} d B_{u} \text { with } h_{t}=1-\rho(T-t) \\
M_{t}^{2} & =-\sqrt{1-\rho^{2}} \int_{0}^{t}(T-u) d \beta_{u} \\
A_{t} & =\int_{0}^{t}\left(\frac{W_{u}^{Y}}{h_{u}}\right)^{+} d\left\langle M^{1}\right\rangle_{u} .
\end{aligned}
$$

For simplicity we fix $\bar{T}=2$. We can conclude using Theorem 4.2 that (NFLVRS) holds true:

(1) If $\rho \leq 0$, then $h>0$ and conditions (C1) and (C2) are trivially satisfied with $\tilde{a}^{2} \equiv 0$. Condition (C3) also holds true.

(2) If $\rho>0$ then, $\left\{(\omega, t) \mid h_{t} \leq 0\right\}=[0, \max (0,2-1 / \rho)]$. For $\rho \in(1 / 2,1]$, this interval is not empty and $d A_{t}^{2}>0$ whenever $W_{t}^{Y}<0$, and these negative excursions of $W^{Y}$ occur a.s. on every bounded interval. Hewever, there are no arbitrage opportunities in this case neither: all conditions are fulfilled to construct the fundamental supermartingale measure $\mathbb{P}^{*}$.

5.4. Filtering models with vanishing noise. Another class of examples fitting in the framework of converging prices are filtering models where the noise in the observation process is vanishing at a fixed time $T$.

We consider two price processes corresponding to the same contingent claim $\xi$. On one market, the price is given by $X$, where, as in the previous example, $X$ is a $\mathbb{P}$-Brownian motion starting at $X_{0}$ and $\xi=X_{T}$. On the other market, investors have access to a noisy observation of $X$. More precisely, we assume that investors on the second market observe the following process:

$$
O_{t}=\int_{0}^{t} f\left(X_{s}\right) d s+W_{t}
$$

$W$ being a Brownian motion independent from $X$. Furthermore, at time $T$ the value $\xi=$ $X_{T}$ can be observed fully. More precisely, the information available for the investors on the second market, denoted by $\mathbb{H}:=\left(\mathcal{H}_{t}\right)_{t \in[0, T]}$ is given by:

$$
\begin{aligned}
\mathcal{H}_{t} & =\sigma\left(O_{s}, s \leq t\right), \text { for } t<T \\
\mathcal{H}_{T} & =\sigma\left(O_{s}, s \leq T\right) \vee \sigma(\xi) .
\end{aligned}
$$


We denote $\mathcal{G}_{t}=\sigma\left(X_{s}, s \leq t\right) \vee \mathcal{H}_{t}$. Now, we assume that:

$$
\left.Y_{t}:=\mathbb{E}\left[\xi \mid \mathcal{H}_{t}\right] \quad \text { (obviously } Y_{T}=\xi\right) \text {. }
$$

We denote by $N_{t}=O_{t}-\int_{0}^{t} \widehat{f\left(X_{s}\right)} d s$ the innovation process, where as usual $\widehat{f(X)}$ is the $\mathbb{H}$-optional projection of the process $f(X)$. We obtain for some $\mathbb{F}^{Y}$-predictable process $\psi$ and for $t<T$ :

$$
Y_{t}=\mathbb{E}\left[X_{t} \mid \mathcal{F}_{t}^{Y}\right]=X_{0}+\int_{0}^{t} \psi_{u} d N_{u}
$$

The process $\psi$ is given by: $\psi_{t}=\widehat{f\left(X_{t}\right) X_{t}}-\widehat{X_{t}} \widehat{f\left(X_{t}\right)}$ (see Theorem 3.35 in [4]). In the filtration $\mathbb{G}$, replacing $N_{t}=W_{t}+\int_{0}^{t}\left(f\left(X_{s}\right)-\widehat{f\left(X_{s}\right)}\right) d s$ we obtain the following representation:

$$
\begin{aligned}
Y_{t} & =X_{0}+\left(\int_{0}^{t} \psi_{u}\left(f\left(X_{u}\right)-\widehat{f\left(X_{u}\right)}\right) d u+\mathbb{1}_{\{t \geq T\}}\left(\xi-Y_{T-}\right)\right)+\int_{0}^{t} \psi_{u} d W_{u} \\
& =X_{0}+V_{t}^{Y}+M_{t}^{2} .
\end{aligned}
$$

Here we have an example with $M^{1} \equiv 0$. We can use Theorem 3.4 to deduce that the dynamics of $Y$ is not compatible with (NFLVRS): we can write

$$
V_{t}^{Y}=J_{t}^{Y}+\left\{\int_{0}^{t} \psi_{u}\left(f\left(X_{u}\right)-\widehat{f\left(X_{u}\right)}\right) d u\right\}
$$

where $J_{t}^{Y}=\mathbb{1}_{\{t \geq T\}}\left(\xi-Y_{T-}\right)$ is not a nonincreasing process.

\section{APPENDIX A. SOME RECALLS ON MEASURES AND INCREASING PROCESSES}

For the reader's convenience we gather here some elementary results that were used in the paper.

Theorem A.1. Let $\mu^{1}$ and $\mu^{2}$ be two finite (possibly signed) measures.

(a) Assume that both $\mu^{1}$ and $\mu^{2}$ are positive measures. Then, $\left(\mu^{1}-\mu^{2}\right)$ is a positive measure only if $\mu^{2}$ is absolutely continuous with respect to $\mu^{1}$.

(b) Assume that $\mu^{1} \perp \mu^{2}$ and furthermore $\left(\mu^{1}+\mu^{2}\right)$ is a positive measure. Then, both $\mu^{1}$ and $\mu^{2}$ are positive measures.

An increasing process can be seen as a random measure on $\mathbb{R}^{+}, d A_{t}(\omega)$, whose distribution function is $A_{\bullet}(\omega)$. Similarly, a process of finite variation can be seen as a signed random measure, since it can be written as the difference of two increasing processes.

Proposition A.2 ([17] p.30). Let A, B be finite variation processes (resp. increasing processes) such that $d B \ll d A$. Then, there exists an optional (resp. nonnegative) process $H$ such that $B=\int H d A$ up to an evanescent set. If moreover $A$ and $B$ are predictable, one may choose $H$ to be predictable. 
Proposition A.3 ([9]). Let $A, B$ be càdlàg, predictable processes of finite variation, with $B$ being increasing. Then, there is a predictable process $\varphi$ and a predictable subset $N$ of $\mathbb{R}_{+} \times \Omega$ such that:

$$
A=\int \varphi d B+\int \mathbb{1}_{N} d A
$$

and:

$$
\int_{\mathbb{R}_{+}} \mathbb{1}_{N}(u) d B_{u}=0 .
$$

Acknowledgements. We are very indebted to Martin Schweizer and two anonymous referees for the careful reading and many suggestions that helped improve our paper.

The research of Monique Jeanblanc is supported by Chair Markets in Transition (Fédération Bancaire Française) and Labex ANR 11-LABX-0019.

\section{REFERENCES}

[1] Aksamit, A. And M. Jeanblanc: Enlargement of Filtration with Finance in View, Springer brief (2017).

[2] ANSEl, J.-P. AND C. STRICKER: Lois de martingale, densités et décomposition de Föllmer Schweizer. Annales de l'I. H. P., section B, tome 28(3) 375-392 (1992).

[3] BACK, K.: Asset Pricing for General Processes. Journal of Mathematical Economics, vol. 20, issue 4, 371-395 (1991).

[4] BAIN, A. AND D. CRISAN: Fundamentals in stochastic filtering, Springer New York (2009).

[5] P. BrÉmaud, M. Yor: Changes of filtration and of probability measures, Z.f.W, 45, 269-295 (1978).

[6] Choulli, T. And C. STRICKer: Deux applications de la décomposition de Galtchouk-KunitaWatanabe, Séminaire de Probabilités XXX, 12-23, Springer (1996).

[7] Dellacherie, C.: Capacités et processus stochastiques, Springer-Verlag (1972).

[8] Delbaen, F. AND W. Schachermayer: A general version of the fundamental theorem of asset pricing. Math. Ann. 300, 463-520 (1994).

[9] Delbaen, F. AND W. SChaChermayer: The existence of absolutely continuous local martingale measures. Annals of Applied Probability 5(4), 926-945 (1995).

[10] Delbaen, F. AND W. SChachermayer: The fundamental theorem for unbounded stochastic processes. Math. Ann. 312, 215-250 (1998).

[11] Elliott, R.J. and Jeanblanc, M. And M. Yor: On models of default risk, Math. Finance, 10, 179-196, (2000).

[12] Föllmer, H. AND M. SchweIZER: Hedging of contingent claims under incomplete information in: M. H. A. Davis and R. J. Elliott (eds.), Applied Stochastic Analysis, Stochastics Monographs, Vol.5, Gordon and Breach, London, 389-414 (1991).

[13] Föllmer, H. And M. SchweIZer: The Minimal Martingale Measure in: R. Cont (ed.), "Encyclopedia of Quantitative Finance", Wiley, 1200-1204 (2010).

[14] Frittelli, M.: Semimartingales and asset pricing under constraints, in Mathematics of Derivative Securities (Cambridge, 1995). Publications of the Newton Institute 15 265-277. Cambridge Univ. Press, Cambridge.

[15] He, S., J. WAng And J. YAn : Semimartingale Theory and Stochastic Calculus. Science Press and CRC Press (1992). 
[16] JARrow, R., P. PRotTER AND S. Pulido: The effect of trading futures on short sale constraints. Mathematical Finance. doi: 10.1111/mafi.12013 (2012).

[17] JACOD, J. AND A. N. ShiryaEV: Limit theorems for stochastic processes, Springer. Second edition (2003).

[18] Jeulin, Th. And Yor, M.: Inégalité de Hardy, semimartingales et faux-amis, Séminaire de Probabilités XIII, 332-359, Lecture Notes in Mathematics, 721 (1979).

[19] JouInI, E. AND H. KALlAL: Arbitrage in securities markets with short-sales constraints. Math. Finance, 5, 197-232 (1995).

[20] KARATZAS, I. AND C. KARDARAS: The numéraire portfolio in semimartingale financial models. Finance Stoch. 11 447-493 (2007).

[21] Meyer, P. A.: Probabilités et potentiel, Paris: Hermann, (1966).

[22] NAPP, C.: The Dalang-Morton-Willinger theorem under cone constraints. J. Math. Econom. 39 111126 (2003).

[23] Nikeghbali, A.: An essay on the general theory of stochastic processes. Probability Surveys Vol. 3, 345-412 (2006).

[24] Protter, P.E.: Stochastic integration and differential equations. Springer. Second edition (2005), version 2.1.

[25] Pham, H. And N. TouzI: The fundamental theorem of asset pricing with cone constraints. J. Math. Econom. 31, 265-279 (1999).

[26] Pulido, S.: The fundamental theorem of asset pricing, the hedging problem and maximal claims in financial markets with short sales prohibitions, Annals of Applied Probability, Vol. 24, No. 1, 54-75 (2014).

[27] SchweIZER, M.: Martingale Densities for General Asset Prices, Journal of Mathematical Economics 21, 363-378 (1992).

[28] SCHWEIZER, M.: On the minimal martingale measure and the Föllmer-Schweizer decomposition, Stochastic Analysis and Applications 13, 573-599 (1995).

University of Zurich, Departement of Banking And Finance, Plattenstrasse 32, ZÜrich 8032, SWITZERLAND.

E-mail address: delia.coculescu@bf.uzh.ch

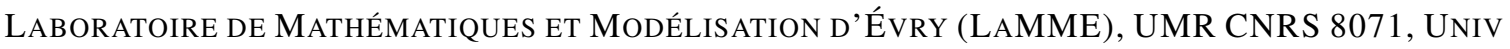
EVRY, UNIVERSITÉ PARIS SACLAY, .

E-mail address: monique. jeanblanceuniv-evry. fr 